hep-th/0409314

\title{
Stability and Thermodynamics of AdS Black Holes with Scalar Hair
}

\author{
Thomas Hertog ${ }^{1}$ and Kengo Maeda ${ }^{2}$ \\ 1 Department of Physics, UCSB, Santa Barbara, CA 93106 \\ 2 Department of General Education, Kobe City College of Technology, \\ 8-3 Gakuen-higashi-machi, Nishi-ku, Kobe 651-2194, Japan
}

\begin{abstract}
Recently a class of static spherical black hole solutions with scalar hair was found in four and five dimensional gauged supergravity with modified, but AdS invariant boundary conditions. These black holes are fully specified by a single conserved charge, namely their mass, which acquires a contribution from the scalar field. Here we report on a more detailed study of some of the properties of these solutions. A thermodynamic analysis shows that in the canonical ensemble the standard Schwarzschild-AdS black hole is stable against decay into a hairy black hole. We also study the stability of the hairy black holes and find there always exists an unstable radial fluctuation, in both four and five dimensions. We argue, however, that Schwarzschild-AdS is probably not the endstate of evolution under this instability.
\end{abstract}

\footnotetext{
${ }^{1}$ Hertog@vulcan.physics.ucsb.edu

${ }^{2}$ kmaeda@kobe-kosen.ac.jp
} 


\section{Introduction}

The original no hair theorem of Bekenstein [1] proves there are no asymptotically flat black hole solutions with scalar hair for minimally coupled scalar fields with convex potentials. This was generalized to minimally coupled scalars with arbitrary positive potentials in [2. But the no scalar hair theorem does not hold, in general, if the scalar field asymptotically tends to a local extremum of the potential [3]. In asymptotically flat space, this obviously requires potentials with negative regions. While potentials of this type arise generically as effective four dimensional potentials in Calabi-Yau compactifications [4, the observation of [3] also leads to asymptotically anti-de Sitter (AdS) space as a natural context to study hairy black holes.

Static, spherically symmetric asymptotically AdS black holes with scalar hair were found (for minimal coupling) analytically in three dimensions [5], and numerically in four [6, 7, 8] and five [8] dimensions. In four dimensions, a class of hairy black holes that are asymptotically locally AdS was found in 9. In most of these examples the scalar field asymptotically goes to a negative maximum of the potential, but the scalar field mass is generally taken to satisfy the Breitenlohner-Freedman (BF) bound [10]. For certain boundary conditions, this ensures that the AdS solution itself is nonlinearly stable provided the potential is of the form $V=(D-2) W^{2}-(D-1) W^{2}$ in $D$ dimensions [11. However, the asymptotic conditions obeyed by all known hairy black hole solutions do not belong to this class. Although they preserve AdS invariance [5, 8, 12, they render the AdS vacuum nonlinearly unstable [13].

Nevertheless, the hairy black holes found in [8] are solutions of a consistent truncation of $\mathcal{N}=8$ gauged supergravity in four and five dimensions. These theories are thought to arise as the low energy limit of $\mathrm{M} /$ string theory with boundary conditions $A d S_{4} \times S^{7}$ and $A d S_{5} \times S^{5}$. For those boundary conditions we have the celebrated AdS/CFT correspondence [14, which provides a non-perturbative definition of string theory on asymptotically AdS spacetimes in terms of a conformal field theory (CFT). In four dimensions, the hairy black holes obey unusual, but AdS invariant asymptotic conditions, which deform the usual dual $2+1$ CFT on a stack of M2 branes by a triple trace operator $[8,15]$. The hairy black holes are described by approximately thermal states in this deformed dual field theory. AdS/CFT relates the thermodynamics of AdS black holes to the thermodynamics of the dual CFT [16], providing 
a novel way to study phase transitions in field theories. Vice versa, one can hope to use the AdS/CFT correspondence to better understand the microscopic description of the hairy black holes. In particular, it is an interesting question how string theory distinguishes between a hairy black hole and Schwarzschild-AdS of the same mass, which is a solution too for the same boundary conditions.

With these general motivations in mind, we now turn to a more detailed study of the hairy black holes found in 8 .

\section{Hairy black holes in $D=4$ Supergravity}

We consider four dimensional gravity minimally coupled to a scalar field with action

$$
S=\int d^{4} x \sqrt{-g}\left[\frac{1}{2} R-\frac{1}{2}(\nabla \phi)^{2}+2+\cosh (\sqrt{2} \phi)\right]
$$

where we have set $8 \pi G=1$. This is a consistent truncation of $\mathcal{N}=8$ gauged supergravity in four dimensions [17, which is the low energy limit of string theory with $A d S_{4} \times S^{7}$ boundary conditions. The potential has a global negative maximum at $\phi=0$, where the scalar has mass (note that $l_{a d s}^{2}=1$ )

$$
m^{2}=-2
$$

This is slightly above the Breitenlohner-Freedman bound [10],

$$
m_{B F}^{2}=\frac{-(D-1)^{2}}{4}
$$

in $D=4$ dimensions, which ensures the perturbative stability of the $A d S$ solution. Here we are interested in nonlinear perturbations of AdS. In particular we will consider the class of asymptotically anti-de Sitter solutions specified by the following set of asymptotic conditions

$$
\begin{gathered}
\phi\left(r, t, x^{a}\right)=\frac{\alpha\left(t, x^{a}\right)}{r}+\frac{c \alpha^{2}\left(t, x^{a}\right)}{r^{2}} \\
g_{r r}=\frac{1}{r^{2}}-\frac{\left(1+\alpha^{2} / 2\right)}{r^{4}}+\frac{M_{0}\left(t, x^{a}\right)}{r^{5}} \\
g_{t r}=O\left(1 / r^{2}\right) \\
g_{r a}=O\left(1 / r^{2}\right)
\end{gathered}
$$


where $x^{a}=\theta, \phi$, and $c$ is an arbitrary constant that labels a one-parameter class of different boundary conditions. For all values of $c$ this set of asymptotic conditions on the fields preserves the full AdS symmetry group 8, despite the fact that the asymptotic behavior of certain metric components is generally relaxed, compared to the standard set of AdS invariant boundary conditions [18. The conserved charges that generate the AdS symmetries are well-defined and finite for all $c$, but acquire a contribution from the scalar field.

Spherically symmetric solutions can be written as

$$
d s^{2}=-f e^{-2 \delta} d t^{2}+f^{-1} d r^{2}+r^{2} d \Omega^{2}
$$

where $f(t, r)$ and $\delta(t, r)$. The field equations reduce to

$$
\begin{gathered}
(1-f)-r f^{\prime}=r^{2}\left[\frac{1}{2} f^{-1} e^{2 \delta} \dot{\phi}^{2}+\frac{f}{2} \phi^{\prime 2}+V(\phi)\right], \\
\dot{f}=-r f \dot{\phi} \phi^{\prime}, \\
2 \delta^{\prime}=-r\left[f^{-2} e^{2 \delta} \dot{\phi}^{2}+\phi^{\prime 2}\right], \\
-\left(e^{\delta} f^{-1} \dot{\phi}\right)+\frac{1}{r^{2}}\left(r^{2} e^{-\delta} f \phi^{\prime}\right)^{\prime}=e^{-\delta} \frac{d V}{d \phi},
\end{gathered}
$$

where $\dot{\phi}=\partial_{t} \phi$ and $\phi^{\prime}=\partial_{r} \phi$.

In [8] it was shown that the theory (2.1) with boundary conditions (2.4)-(2.5) and $c \neq 0$ admits a one-parameter class of regular static, spherically symmetric black hole solutions with scalar hair outside the horizon. We summarize the results of [8] in Figure 1, where we plot the value $\phi_{e}$ of the field at the horizon as a function of horizon size $R_{e}$, for two different choices of AdS invariant boundary conditions, namely $c=-1$ (bottom) and $c=-1 / 4$ (top). The hairy black hole solutions are specified by a single conserved charge, their mass, which is given by

$$
E_{h}=4 \pi\left(M_{0}+\frac{4}{3} c \alpha^{3}\right)
$$

where the second term is a finite scalar contribution. The mass was computed in [8], where it was shown that $E_{h} \sim R_{e}^{3}$ for large $R_{e}$. For a given horizon size $R_{e}$ one has $E_{h}>E_{s}=4 \pi\left(R_{e}+R_{e}^{3}\right)$. For large $R_{e}$ it was found (see Figure 10 in [8]) that $E_{h} \rightarrow E_{s}$, which means $E_{h}=4 \pi R_{e}^{3}\left(1+\mathcal{O}\left(1 / R_{e}\right)\right)$ in this regime. On the other hand, 
since Schwarzschild-AdS is a solution too for all $c$, one has two very different black hole solutions for a given mass. The hairy black holes thus provide an example of black hole non-uniqueness.

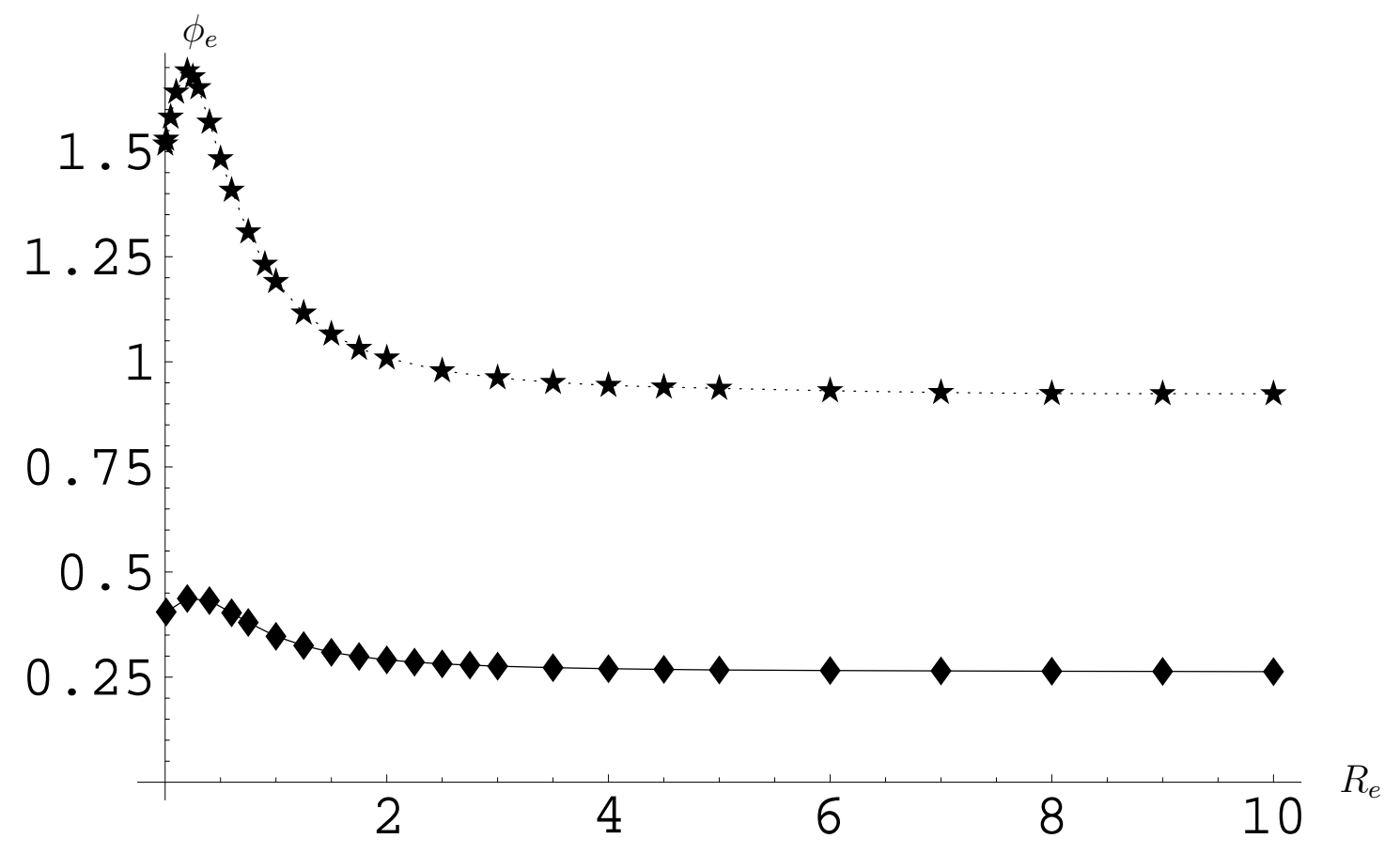

Figure 1: The scalar field $\phi_{e}$ at the horizon as a function of horizon size $R_{e}$ in hairy black hole solutions of $D=4$ gauged supergravity. The two curves correspond to solutions with two different AdS invariant boundary conditions, labelled by $c=-1$ (bottom) and $c=-1 / 4$ (top).

\section{$3 \quad$ Stability}

We now consider linear radial fluctuations around the hairy black hole background. Substituting

$$
\begin{aligned}
\phi(r, t) & =\phi_{0}(r)+\phi_{1}(r) e^{i \omega t} \eta \\
f(r, t) & =f_{0}(r)+f_{1}(r) e^{i \omega t} \eta \\
\delta(r, t) & =\delta_{0}(r)+\delta_{1}(r) e^{i \omega t} \eta
\end{aligned}
$$

in the field equations (where $\left(f_{0}(r), \delta_{0}(r), \phi_{0}(r)\right)$ are the background quantities) and expanding in $\eta$ yields, to first order, the following perturbation equation for the scalar 
field,

$$
\frac{e^{2 \delta_{0}}}{f_{0}} \frac{d^{2} \phi_{1}}{d r^{* 2}}+\frac{2 f_{0}}{r} \phi_{1}^{\prime}+\left[e^{2 \delta_{0}} f_{0}^{-1} \omega^{2}-2 f_{0}{\phi_{0}^{\prime}}^{2}-\frac{r^{2}}{2} f_{0} \phi_{0}^{\prime 4}-\left(r \phi_{0}^{\prime} f_{0}\right)^{\prime}\right] \phi_{1}=V_{, \phi \phi}\left(\phi_{0}\right) \phi_{1},
$$

where $d r^{*} / d r=e^{\delta_{0}} / f_{0}$. Eliminating $\phi_{0}^{\prime \prime}$ using eq. (2.10) and defining $\chi=r \phi_{1}$, one obtains

$$
-\frac{d^{2} \chi}{d r^{* 2}}+P \chi=\omega^{2} \chi
$$

with

$$
P=f_{0} e^{-2 \delta_{0}}\left(-\frac{1}{2}\left({\phi_{0}^{\prime}}^{2}+r^{2} \phi_{0}^{\prime 4}\right) f_{0}+\left(\frac{1}{r}-r{\phi_{0}^{\prime}}^{2}\right) f_{0}^{\prime}+2 r \phi_{0}^{\prime} V_{, \phi}\left(\phi_{0}\right)+V_{, \phi \phi}\left(\phi_{0}\right)\right) .
$$

A hairy black hole is unstable if there exists a solution to (3.3) with $\omega^{2}<0$, since small fluctuations of this kind grow exponentially in time.

One immediately sees that at the horizon the potential $P$ vanishes. Near spacelike infinity on the other hand one has, for general $\mathrm{m}^{2}$,

$$
P \rightarrow\left(m^{2}-m_{c}^{2}\right) e^{-2 \delta_{0}} r^{2}
$$

where $m_{c}^{2}=-D(D-2) / 4$ is the conformal mass. Thus $P$ is unbounded from below for scalars with mass $m^{2}<m_{c}^{2}$. The fact that the conformal mass separates two qualitatively different regimes is true in any dimension. For $m^{2}=m_{c}^{2}$, which is the case of interest to us, the subleading terms are important at large $r$, giving

$$
P \rightarrow \frac{-\alpha^{2}}{2} e^{-2 \delta_{0}}
$$

Therefore, $P$ tends to a negative constant in our case, for all choices of boundary conditions. In Figure 2 we show the potential for perturbations around a hairy black hole of size $R_{e}=2$, in two different theories, specified by $c=-1$ and $c=-1 / 4$.

The fact that $P$ is asymptotically negative means there exist exponentially growing fluctuations, potentially causing the hairy black holes to be unstable. It only remains to verify there exists an unstable mode that obeys the modified boundary conditions with the same value of $c$ as the black hole background. Near the horizon we have $r^{*} \rightarrow-\infty$ and $P \rightarrow 0$, so the general near horizon solution of (3.3) is given by

$$
\chi=a e^{\sigma r^{*}}+b e^{-\sigma r^{*}}
$$



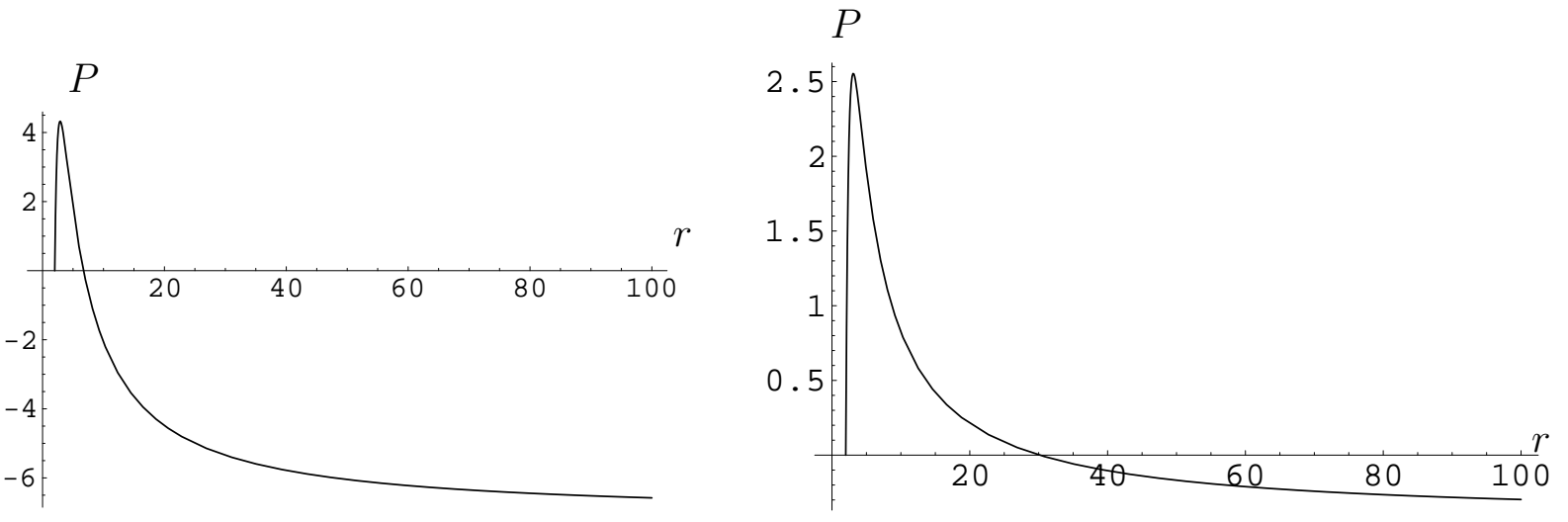

Figure 2: Potential for linearized radial fluctuations around a hairy black hole with radius $R_{e}=2$, for $c=-1 / 4$ (left) and $c=-1$ (right) boundary conditions.

where $\omega=-i \sigma$. Regularity at the horizon requires $b=0$, since future unstable modes have $\sigma>0$. This translates into the following boundary condition in terms of the original $r$ coordinate. From (2.7) and (2.10) it follows

$$
\left.\kappa \equiv f_{, r}\right|_{R_{e}}=\frac{1-R_{e}^{2} V\left(\phi\left(R_{e}\right)\right)}{R_{e}}
$$

Since $f \simeq \kappa\left(r-R_{e}\right)$ near the horizon, this gives

$$
r^{*} \simeq \frac{e^{\delta_{0}}}{\kappa} \ln \left(r-R_{e}\right)=\frac{\ln \left(r-R_{e}\right)}{\kappa},
$$

where we have set $\delta_{0}\left(R_{e}\right)=0$. Hence (3.7) becomes

$$
\chi=a\left(r-R_{e}\right)^{\frac{\sigma}{\kappa}}
$$

giving, at $\epsilon=\left(r-R_{e}\right)$,

$$
\frac{d \chi}{d r}(\epsilon)=\frac{\sigma \chi(\epsilon)}{\kappa \epsilon}
$$

Finally, to satisfy (2.4) with the same value of $c$ as the background, we must require that near spacelike infinity the fluctuations behave as

$$
\phi_{1}(r)=\frac{\chi}{r}=\left(\frac{\alpha_{1}}{r}+\frac{2 c \alpha_{0} \alpha_{1}}{r^{2}}\right) .
$$

Here $\alpha_{0}$ is the coefficient of the $1 / r$ mode of the background solution, $\phi_{0}$, and $\alpha_{1}$ is an arbitrary constant. Now, for large $r$, equation (3.3) for the fluctuations is approximately

$$
-\frac{d^{2} \chi}{d r^{* 2}}=\left(\omega^{2}-\bar{p}\right) \chi=\tilde{\omega}^{2} \chi
$$




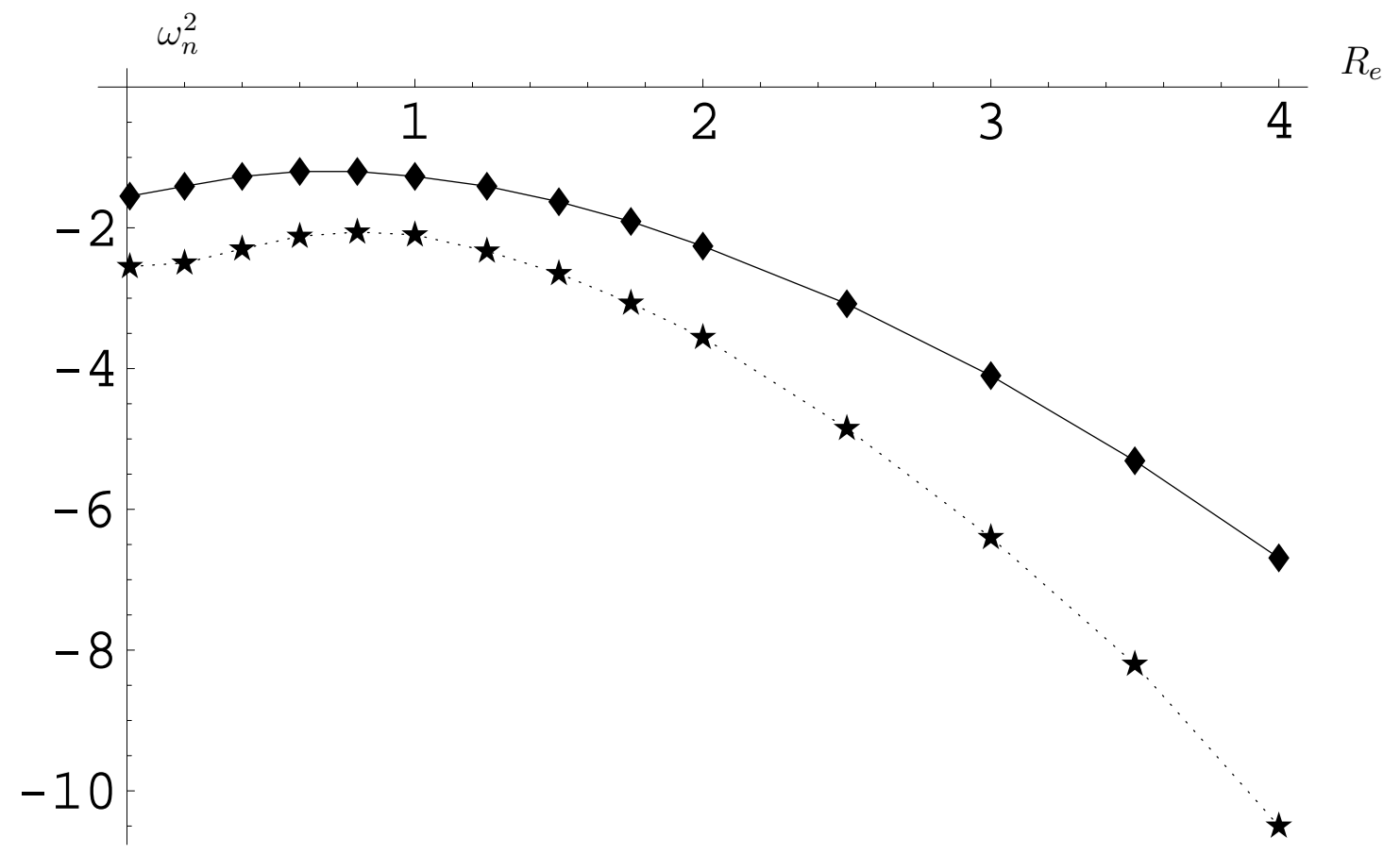

Figure 3: The value of the negative frequency, $\omega_{n}^{2}$, of the unstable radial perturbation as a function of horizon size $R_{e}$, in $D=4$ supergravity with two different AdS invariant boundary conditions, namely $c=-1$ (bottom) and $c=-1 / 4$ (top).

In the frequency regime of interest, $\bar{p}<\omega^{2}<0$, this yields

$$
\chi=A \cos \left(\tilde{\omega} r^{*}+\gamma\right) \simeq A \cos (-\tilde{\omega} / r+k \tilde{\omega}+\gamma) \sim A\left(\cos (k \tilde{\omega}+\gamma)+\frac{\tilde{\omega}}{r} \sin (k \tilde{\omega}+\gamma)\right),
$$

where we used $r^{*} \simeq-1 / r+k$ with $k$ a constant. Therefore, the constraint (3.12) translates into the following condition on the frequency $\tilde{\omega}$,

$$
\tan (k \tilde{\omega}+\gamma)=\frac{2 c \alpha_{0}}{\tilde{\omega}} .
$$

For given $\omega^{2}$, the boundary conditions at the horizon uniquely determine the solution for the rescaled fluctuation $\chi(r)$. But we find that for all horizon sizes $R_{e}$, there is precisely one negative frequency, $\omega_{n}^{2}$, for which the asymptotic condition (3.15) is satisfied too. Our results are shown in Figure 3, where we plot the value of this frequency, $\omega_{n}^{2}$, as a function of the black hole radius $R_{e}$, in two different theories. For large $R_{e}$, we find $\omega_{n}^{2} \propto R_{e}^{2}$, which is natural since $\bar{p} \propto \alpha^{2} \propto R_{e}^{2}$ (the last step follows 
from the results in [8]). Hence we conclude that the hairy black holes found in [8] are unstable to linearized radial fluctuations exponentially growing in time.

\section{Thermodynamics}

The canonical ensemble is defined by a Euclidean path integral over matter fields and metrics which tend asymptotically respectively to zero and AdS space, identified periodically in $\tau=i t$ with period $\beta$. The inverse of the period $\beta$ corresponds to the temperature $T$. The path integral is usually approximated by summing over saddlepoints. Periodically identified AdS space is one of these and we take it to be the zero of action and energy. This gives the dominant contribution at low temperature, but it is well known [19] that at sufficiently high temperature a large Euclidean SchwarzschildAdS black hole solution has lower action. In this regime the pure radiation will then tend to tunnel to the black hole configuration at a rate determined by the difference of the actions of both geometries.

Here we consider a canonical ensemble described by a path integral with boundary conditions given by (2.4) and (2.5) for some $c$. The Euclidean continuation of the hairy black hole solution gives an extra saddle-point contribution. This raises the question whether there is a temperature regime where Schwarzschild-AdS is likely to 'decay' into a hairy black hole, hereby spontaneously acquiring a nontrivial scalar field profile outside its horizon.

To study this issue it is sufficient to consider the minisuperspace consisting of static, spherically symmetric Euclidean metrics,

$$
d s^{2}=e^{-2 \delta(r)} f(r) d \tau^{2}+f^{-1}(r) d r^{2}+r^{2} d \Omega^{2},
$$

where $0 \leq t \leq \beta, r \geq R_{e}$ and the scalar field $\phi(r)$. The reduced Hamiltonian action reads,

$$
\begin{aligned}
I & =\int N \mathcal{H}+B \\
& =4 \pi \beta \int_{R_{e}}^{\infty} e^{-\delta} r^{2}\left[\frac{f^{\prime}}{r}-\frac{1}{r^{2}}(1-f)+\frac{1}{2} f \phi^{\prime 2}+V(\phi)\right] d r+B
\end{aligned}
$$

where

$$
\delta B=-4 \pi \beta e^{-\delta}\left[r \delta f+r^{2} f \phi^{\prime} \delta \phi\right]_{R_{e}}^{\infty}
$$


The variation of the boundary term is determined by the condition that the action is an extremum under the variation of the fields considered here [18, 20]. The last term in (4.3) comes from the scalar field variation. The geometries in the variation are smooth and complete if and only if the period $\beta$ satisfies

$$
\beta e^{-\delta\left(R_{e}\right)} f^{\prime}\left(R_{e}\right)=4 \pi
$$

We first consider the contribution from the Euclidean continuation of the hairy black hole solution (2.6) . For this, (4.4) gives the temperature as a function of horizon size $R_{e}$

$$
2 \pi T=\frac{2 \pi}{\beta}=\frac{1-V\left(\phi\left(R_{e}\right)\right) R_{e}^{2}}{2 R_{e}}
$$

One sees that, like Schwarzschild-AdS, the hairy black hole only contributes to the thermodynamic ensemble at high temperatures, $2 \pi T \geq \sqrt{-V\left(\phi\left(R_{e}\right)\right)}$. For a given (sufficiently high) temperature, there are two possible hairy black hole masses that can be in equilibrium with thermal radiation. The lower of these has negative specific heat so it is unstable to decay into pure thermal radiation. The higher mass black hole, by contrast, has always positive specific heat (and smaller action) so we concentrate on this.

For the asymptotic conditions on the fields defined in Section 2, both the gravitational and scalar sector give divergent contributions to the variation of the boundary term (4.3) at infinity. However, it was shown in 8] (and in a Euclidean setting in [9, 13.) that the divergences cancel out, yielding a finite total surface term at infinity,

$$
\left.B\right|_{\infty}=\beta E_{h}
$$

At the horizon we have

$$
\left.\delta B\right|_{R_{e}}=\left.4 \pi \beta e^{-\delta\left(R_{e}\right)} R_{e} \delta f\right|_{R_{e}}
$$

Using

$$
\left.\delta f\right|_{R_{e}}=-f^{\prime}\left(R_{e}\right) \delta R_{e}
$$

this gives, upon integration,

$$
\left.B\right|_{R_{e}}=-4 \pi \int\left(\beta e^{-\delta\left(R_{e}\right)} f\left(R_{e}\right)\right) R_{e} \delta R_{e}=-16 \pi^{2} \int R_{e} \delta R_{e}=-8 \pi^{2} R_{e}^{2},
$$


where $\delta R$ is the variation along the horizon. Combining (4.6) and (4.9) and taking into account the Hamiltonian constraint $\mathcal{H}=0$, one obtains

$$
I_{h}=\beta E_{h}-8 \pi^{2} R_{h}^{2}=\beta\left(E_{h}-S T\right)=\beta F
$$

where $S=\pi R_{e}^{2} / G$ and $F$ are the entropy and free energy, respectively.

On the other hand, for the Euclidean Schwarzschild AdS black hole we have

$$
\delta=0, \quad f=1-\frac{E_{s}}{4 \pi r}+r^{2}, \quad R=r .
$$

The temperature is related to the horizon radius $R_{s}$ in the following way,

$$
2 \pi T=\frac{1+3 R_{s}^{2}}{2 R_{s}}
$$

Therefore, the radius of the Schwarzschild-AdS black hole in the canonical ensemble is smaller compared to the horizon size of the hairy black hole. The Euclidean action is given by

$$
I_{s}=4 \pi\left(\beta\left(R_{s}+R_{s}^{3}\right)-2 \pi R_{s}^{2}\right) .
$$

Using the results of [8] we have numerically computed the difference between the Euclidean actions (4.10) and (4.13),

$$
\Delta I=I_{h}-I_{s}
$$

as a function of temperature ${ }^{3}$ and in two different theories. The result is shown in Figure 4. One sees that the action of the hairy black hole is always larger than that of Schwarzschild-AdS. This means the latter is thermodynamically stable, despite the fact there is no Positive Mass theorem for the set of asymptotic conditions we considered here.

We note that our results differ from those obtained in [9], where a thermodynamical analysis was performed in which the action of asymptotically locally AdS hairy black holes was compared with the action of vacuum black holes with the same asymptotic structure. The important point is that with locally AdS boundary conditions, there is no critical temperature below which black holes don't contribute to the thermodynamic ensemble. In this context, 9] finds indeed that for sufficiently low temperatures, the vacuum black hole is likely to decay into a hairy solution.

\footnotetext{
${ }^{3}$ The curve in the $c=-1 / 4$ theory starts at higher temperature because the hairy black hole solution does not contribute to the thermodynamic ensemble at temperatures below $T=\sqrt{\left|V\left(R_{e}\right)\right|}$.
} 


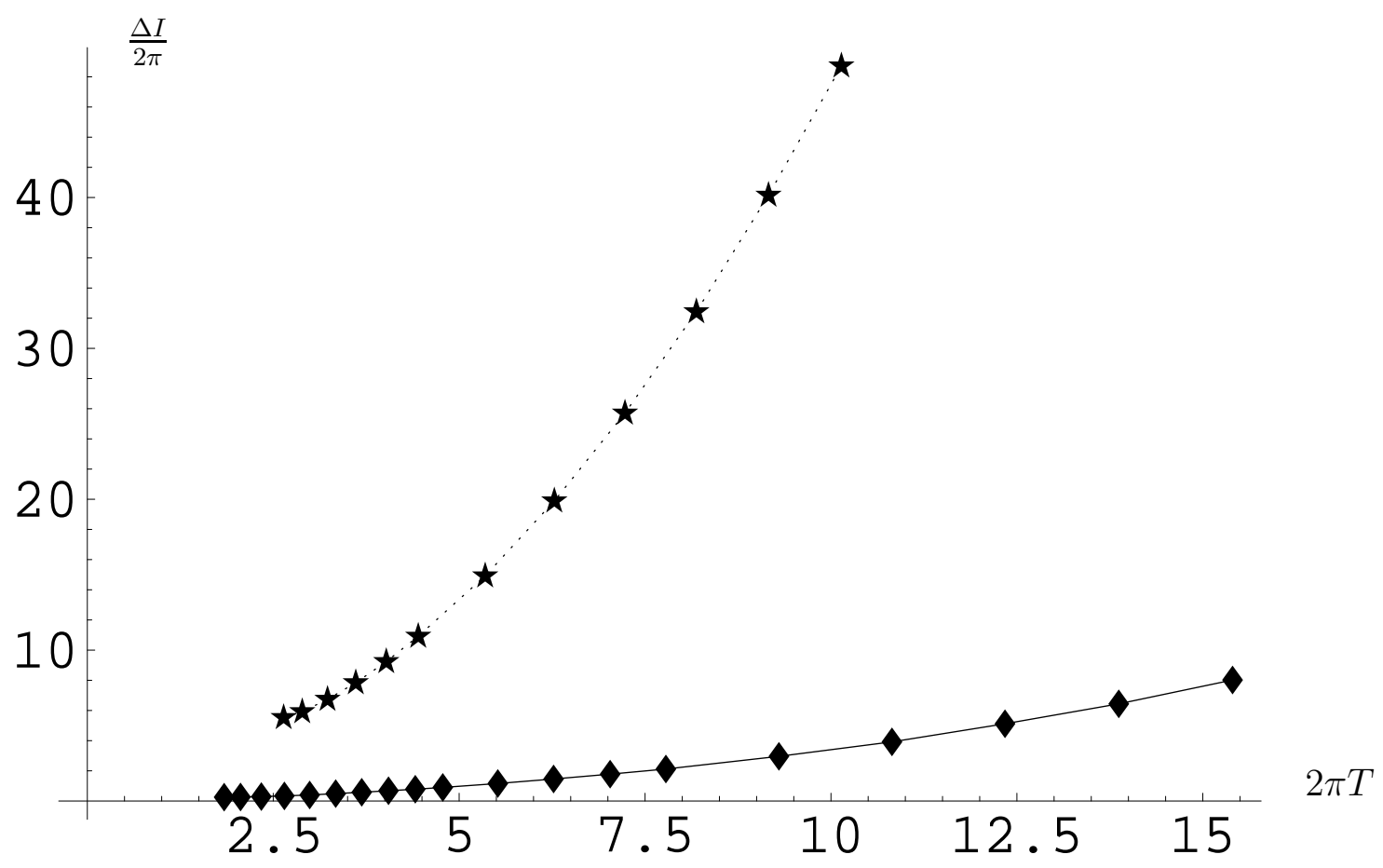

Figure 4: The difference $\Delta I$ between the Euclidean action of a hairy black hole, $I_{h}$, and that of Schwarzschild-AdS, $I_{s}$, as a function of temperature $T$. The two curves correspond to hairy black hole solutions of $D=4$ gauged supergravity with two different AdS invariant boundary conditions, specified by $c=-1$ (bottom) and $c=-1 / 4$ (top). One sees that in both cases Schwarzschild-AdS is always thermodynamically favored.

\section{$5 \quad$ Hairy Black Holes in $D=5$ Supergravity}

Finally, we briefly consider five dimensional gravity minimally coupled to a scalar field with action

$$
S=\int d^{5} x \sqrt{-g}\left[\frac{1}{2} R-\frac{1}{2}(\nabla \phi)^{2}+\left(2 e^{2 \phi / \sqrt{3}}+4 e^{-\phi / \sqrt{3}}\right)\right] .
$$

This is a consistent truncation of $\mathcal{N}=8$ gauged supergravity in five dimensions, which is the low energy limit of string theory with $A d S_{5} \times S^{5}$ boundary conditions. At the global negative maximum of the potential at $\phi=0$, the scalar has mass $m^{2}=-4$ and thus saturates the Breitenlohner-Freedman bound in five dimensions.

In [8] a class of static, spherically symmetric black holes was found with a nontrivial scalar field profile outside the horizon. Asymptotically, the scalar field behaves 
as

$$
\phi(r)=\frac{\alpha_{0}}{r^{2}} \ln r+\frac{\alpha_{0}}{r^{2}}\left(c-\frac{1}{2} \ln \alpha_{0}\right)
$$

where $c$ is a constant that labels the choice of boundary conditions and $\alpha_{0}$ depends on the horizon size. The backreaction of the scalar field causes the $g_{r r}$ component of the metric to fall off slower than usual, but the asymptotic conditions are left invariant under the full AdS symmetry group. The conserved charges generating the symmetries are finite, but acquire a contribution from the scalar field [8, 12]. In particular, the mass of the hairy black holes is given by

$$
E_{h}=2 \pi^{2}\left(\frac{3}{2} M_{0}+\frac{1}{4} \alpha_{0}^{2}\left(\ln \alpha_{0}\right)^{2}+\alpha_{0}^{2}\left(\frac{1}{4}-c\right) \ln \alpha_{0}+\alpha_{0}^{2}\left(c^{2}-\frac{1}{2} c+\frac{1}{8}\right)\right)
$$

where $M_{0}$ is now the $\mathcal{O}\left(r^{-6}\right)$ correction to $g_{r r}$. It is interesting to verify the stability of these hairy black holes, especially since $D=5, \mathcal{N}=8$ supergravity plays a prominent role in the AdS/CFT correspondence. Substituting the following expansions

$$
\begin{aligned}
\phi(r, t) & =\phi_{0}(r)+\frac{\chi(r)}{r^{3 / 2}} e^{i \omega t} \eta, \\
M(r, t) & =M_{0}(r)+M_{1}(r) e^{i \omega t} \eta \\
\delta(r, t) & =\delta_{0}(r)+\delta_{1}(r) e^{i \omega t} \eta
\end{aligned}
$$

in the field equations one obtains, to first order in $\eta$, an equation for the fluctuations of the form (3.3), with

$P(r)=e^{-2 \delta_{0}} f_{0}\left[f_{0}\left(\frac{3}{4 r^{2}}-\frac{5 \phi_{0}^{\prime 2}}{6}-\frac{2 r^{2}}{9} \phi_{0}^{\prime 4}\right)+f_{0}^{\prime}\left(\frac{3}{2 r}-\frac{2 r}{3} \phi_{0}^{\prime 2}\right)+\frac{4 r}{3} \phi_{0}^{\prime} V_{, \phi}\left(\phi_{0}\right)+V_{, \phi \phi}\left(\phi_{0}\right)\right]$.

The potential is unbounded from below, since the scalar mass $m^{2}$ is less than the conformal mass $m_{c}^{2}$. Near spacelike infinity $P$ goes as

$$
P \rightarrow-\frac{1}{4} e^{-2 \delta_{0}} r^{2}
$$

so one expects there should exist an unstable mode. The general asymptotic solution of (5.5) reads

$$
\chi=\frac{\alpha_{1}}{\sqrt{r}} \ln r+\frac{\beta_{1}}{\sqrt{r}}
$$

This belongs to the same class of asymptotically AdS spacetimes as the black hole background itself, provided that

$$
\frac{\beta_{1}}{\alpha_{1}}=\left(-\frac{1}{2} \ln \alpha_{0}+c-\frac{1}{2}\right) .
$$


We find there is always precisely one negative frequency $\omega_{n}^{2}$ for which this condition is satisfied. For instance, for a black hole of radius $R_{e}=1$ in the $c=0$ theory, the unstable mode has frequency $\omega_{n}^{2}=-2.4$.

\section{Discussion}

We have studied the thermodynamics and stability of a class of spherical hairy black holes, which are solutions of $D=4$ gauged supergravity with modified, but AdS invariant boundary conditions. The thermodynamical analysis shows that in the canonical ensemble the usual Schwarzschild-AdS black hole is stable against 'decay' into a hairy black hole. The stability analysis reveals there always exists precisely one unstable radial fluctuation around the hairy black hole background. We find similar results in $D=5$ supergravity.

We should note that the instability of the supergravity hairy black hole solutions is not only a consequence of the modification of the standard AdS boundary conditions, which renders the AdS state itself (nonlinearly) unstable. Rather, it is because the scalar field mass $m^{2}$ is less than or equal to the conformal mass $m_{c}^{2}$ in both cases. Stable hairy AdS black hole exist in theories where $m_{c}^{2}<m^{2}<m_{B F}^{2}+1[6]^{4}$. It would be interesting to find a supergravity example of this.

We can argue that Schwarzschild-AdS is unlikely to be the endstate of the evolution of a perturbed hairy black hole in the following way. Assume for a moment the perturbed hairy black hole does tend to Schwarzschild-AdS and that the decay of the scalar field near the horizon is governed by the lowest quasinormal mode. Then one could approximate the evolution of the scalar field $\phi$ near the horizon as

$$
\phi \simeq \phi_{e} e^{-2 \pi \gamma T\left(v-v_{0}\right)}
$$

where $\gamma$ is a constant and $v=t+r^{*}$. Here we used the fact that the imaginary part of the frequency of quasinormal modes is proportional to the temperature $T$ of Schwarzschild-AdS [21]. Now consider a null geodesic with tangent vector $V^{\mu}$ on the event horizon at radius $R$. Since the affine parameter $\lambda$ is related to $v$ as

$$
\lambda \sim \lambda_{0} e^{2 \pi T\left(v-v_{0}\right)},
$$

\footnotetext{
${ }^{4}$ Although the conventional mass of the solutions presented in 6 diverges, the example with $\alpha=1.55$ in the symmetric double well potential can be reinterpreted as an asymptotically AdS black hole obeying the modified boundary conditions given in 8 .
} 
eq. (6.1) becomes

$$
\phi \sim \phi_{e}\left(\frac{\lambda}{\lambda_{0}}\right)^{-\gamma} .
$$

On the other hand, the Raychaudhuri equation reads

$$
\frac{d \theta}{d \lambda}=-\frac{1}{2} \theta^{2}-T_{\mu \nu} V^{\mu} V^{\nu}
$$

Using

$$
T_{\mu \nu} V^{\mu} V^{\nu}=\dot{\phi}^{2}, \quad \theta=\frac{2 \dot{R}}{R}
$$

this becomes

$$
\ddot{R}=-\frac{\dot{\phi}^{2}}{2} R
$$

where $\dot{\phi}=\partial_{\lambda} \phi$. Since the perturbed hairy black hole must tend to Schwarzschild-AdS of the same total mass, we have (for large initial horizon size $R_{e}$ )

$$
E_{h}=4 \pi R_{e}^{3}\left(1+\mathcal{O}\left(1 / R_{e}\right)\right)=4 \pi\left(R_{f}^{3}+R_{f}\right)
$$

where $R_{f}$ is the radius of the final Schwarzschild-AdS configuration. This means that the final radius $R_{f}$ cannot be a multiple of $R_{e}$, but instead $R_{f}=R_{e}+c_{0}$, where $c_{0}$ is a positive constant. Hence (6.6) can be approximated as follows,

$$
\ddot{R} \simeq-\frac{\dot{\phi}^{2}}{2} R_{e}=-\frac{R_{e}}{2}\left(\frac{\phi_{e} \gamma}{\lambda_{0}}\right)^{2}\left(\frac{\lambda}{\lambda_{0}}\right)^{-2 \gamma-2} .
$$

Integrating yields,

$$
R_{f}=R(\lambda=\infty)=R_{e}\left(1+\frac{\phi_{e}^{2} \gamma}{2(2 \gamma+1)}\right)
$$

But this contradicts the fact that $R_{f}=R_{e}+c_{0}$, since for instance in the $c=-1$ theory one has $\phi_{e} \rightarrow 0.25$ for large $R_{e}$.

This indicates that Schwarzschild-AdS is probably not the endstate of evolution under the instability. Instead, the perturbed black hole is likely to continue to evolve forever, perhaps in a manner envisioned in [22] (in a different context) where the horizon continues to expand. This raises the question whether or not the black hole singularity reaches the boundary of AdS in finite time. If so, small fluctuations would induce a transition between a black hole and a big crunch. The surprising evolution 
under the instability can also be understood from the dual CFT perspective, where the hair turns on the triple trace operator [13]. This indicates that, in contrast to Schwarschild-AdS black holes, the hairy black holes are states concentrated on the unstable side of the field theory potential. The evolution of some states of this kind has been shown before to produce a big crunch singularity [13. One can then hope to use AdS/CFT to shed light on the quantum nature of the spacelike singularities in these dynamical solutions.

\section{Acknowledgments}

We thank T. Torii for helpful discussions. This work was supported in part by NSF grant PHY-0244764.

\section{References}

[1] J. D. Bekenstein, "Exact Solutions of Einstein Conformal Scalar Equations," Ann. Phys. (N.Y.) 82 (1974) 535

[2] M. Heusler, "A No-hair Theorem for Self-gravitating Nonlinear Sigma Models," J. Math. Phys. 33 (1992) 10

[3] D. Sudarsky, J. A. Gonzalez, "On Black Hole Scalar Hair in Asymptotically Anti de Sitter Spacetimes," Phys. Rev. D67 (2003) 024038, gr-qc/0207069

[4] T. Hertog, G. T. Horowitz, K. Maeda, "Negative Energy Density in Calabi-Yau Compactifications," JHEP 0305 (2004) 060, hep-th/0304199

[5] M. Henneaux, C. Martinez, R. Troncoso, J. Zanelli, "Black Holes and Asymptotics of 2+1 Gravity Coupled to a Scalar Field," Phys. Rev. D65 (2002) 104007, hep-th/0201170

[6] T. Torii, K. Maeda and M. Narita, "Scalar hair on the black hole in asymptotically anti-de Sitter spacetime," Phys. Rev. D 64 (2001) 044007

[7] E. Winstanley, "On the Existence of Conformally Coupled Scalar Field Hair for Black Holes in (anti)-de Sitter Space," Found. Phys. 33 (2003) 111, gr-qc/0205092 
[8] T. Hertog, K. Maeda, "Black Holes with Scalar Hair and Asymptotics in $N=8$ Supergravity," JHEP 0407 (2004) 051, hep-th/0404261

[9] C. Martinez, R. Troncoso, J. Zanelli, "Exact Black Hole Solutions with a Minimally Coupled Scalar Field,"

[10] P. Breitenlohner and D. Z. Freedman, "Stability In Gauged Extended Supergravity," Annals Phys. 144 (1982) 249; "Positive Energy In Anti-De Sitter Backgrounds And Gauged Extended Supergravity," Phys. Lett. B 115 (1982) 197

[11] P. K. Townsend, "Positive Energy And The Scalar Potential In Higher Dimensional (Super)Gravity Theories," Phys. Lett. B 148 (1984) 55.

[12] M. Henneaux, C. Martinez, R. Troncoso, J. Zanelli, "Asymptotically Anti-de Sitter Spacetimes and Scalar Fields with a Logarithmic Branch," hep-th/0404236

[13] T. Hertog, G. T. Horowitz, "Towards a Big Crunch Dual," JHEP 0407 (2004) 073, hep-th/0406134

[14] J. M. Maldacena, "The large N limit of superconformal field theories and supergravity," Adv. Theor. Math. Phys. 2 (1998) 231, hep-th/9711200

[15] E. Witten, "Multi-Trace Operators, Boundary Conditions, and AdS/CFT Correspondence," hep-th/0112258

[16] E. Witten, "Anti-de Sitter Space, Thermal Phase Transtion, And Confinement In Gauge Theories," Adv. Theor. Math. Phys. 2 (1998) 505

[17] M. J. Duff, J. T. Liu, "Anti-de Sitter Black Holes in Gauged N=8 Supergravity," Nucl. Phys. B554 (1999) 237, hep-th/9901149

[18] M. Henneaux, C. Teitelboim, "Asymptotically Anti-de Sitter Spaces," Comm. Math. Phys. 98 (1985) 391

[19] S. W. Hawking and D. Page, "Thermodynamics of Black Holes in Anti-de Sitter Space," Commun. Math. Phys. 87 (1983) 577

[20] T. Regge, C. Teitelboim, "Role of Surface Integrals in the Hamiltonian Formulation of General Relativity," Annals Phys. 88 (1974) 286 
[21] G. Horowitz and V. Hubeny, "Quasinormal modes of AdS black holes and the approach to the thermal equilibrium," Phys. Rev. D62 (2000) 024027, hep-th/9909056

[22] M. Alcubierre, J. A. González, M. Salgado, D. Sudarsky, "The Cosmic Censorship Conjecture: Is it Generically Violated?," gr-qc/0402045 